\title{
The Relationship Between the Concepts of Hope and Happiness in Students
}

Leila Ghahremani

Assistant Professor, Department of Health Education and Promotion, School of Health, Shiraz University of Medical Sciences, Shiraz, Iran.

Hadi Tehrani

Assistant Professor, Department of Health Education and Health Promotion, Faculty of Health, Mashhad University of Medical Sciences, Mashhad, Iran.

Social Determinants of Health research center, Mashhad University of Medical Sciences

Abdolhalim Rajabi

Assistant Professor of Epidemiology, faculty of health, Environmental Health Research Center, Golestan University of Medical Sciences, Gorgan, Iran.

Alireza Jafari

PhD Student, Social Determinants of Health Research Center, Gonabad University of Medical Sciences, Gonabad, Iran.

*. PhD Student in Health Education and Health Promotion, Social Determinants of Health Research Center, Mashhad University of Medical Sciences, Mashhad, Iran.( Corresponding Author): Jafari.ar94@gmail.com

Received: 06 September 2019 Accepted: 03 February 2020

Doi: 10.29252/ijhehp.8.2.107

\section{ABSTRACT}

Background and Objectives: Happiness and hope are two concepts that affect students' mental health. The aim of this study was to determine the relationship between the concept of hope and happiness in students.

Materials and Methods: This cross-sectional study was conducted on 250 students in school of health, Shiraz University of Medical Sciences. The data collection tool was Snyder's Hope and MUNSH 's Happiness Questionnaires. Data were analyzed by SPSS version 22 using descriptive and analytical tests including ANOVA, independent samples t-test, Pearson correlation and linear regression.

Results: In this study, the mean ( $\pm S D$ ) of happiness and hope scores were 4.50 $(+0.78)$ and $24.52(+0.66)$ respectively. There was a significant relationship between age $(P=0.001)$ and gender $(P=0.008)$ variables with hope scale. Based on the Pearson correlation coefficient, there was a significant correlation between hope and sub-scales of happiness (Positive affect, negative affect, Positive experience, negative experience) $(P<0.001)$. Results of linear regression analysis showed that, demographic variables, happiness scale and its sub-scales, were able to predict $28.8 \%$ of the variations of hope $(P<0.001)$.

Conclusion: Therefore, it is necessary that the predisposing factors create psychological problems such as disappointment and depression and etc., identified in students and appropriate educational and counseling programs are being designed and implemented.

Keywords: Hope, Happiness, Mental Health, Students

Paper Type: Research Article.

Citation (Vancouver): Ghahremani L, Tehrani H, Rajabi A, Jafari A. The Relationship Between the Concepts of Hope and Happiness in Students. Iran J Health Educ Health Promot. Spring 2020;8(2): 107-117. [Persian]x

$\checkmark$ Citation (APA): Ghahremani L., Tehrani H., Rajabi A., Jafari A. The Relationship Between the Concepts of Hope and Happiness in Students. (Summer 2020). Conc... . Iranian Journal of Health Education \& Health Promotion., 8(2), 107-117 . [Persian] 


\section{ارتباط بين دو مفهوم اميدوارى و شادكامى در دانشجويان}

\section{جكيده}

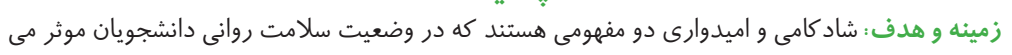

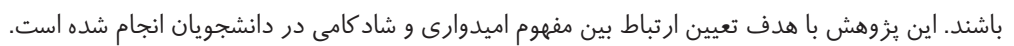

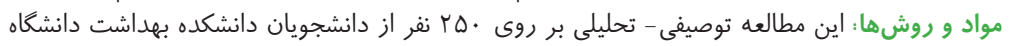

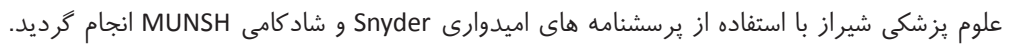

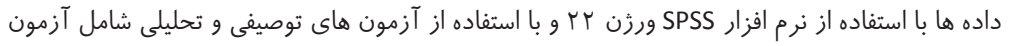

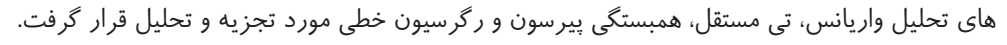

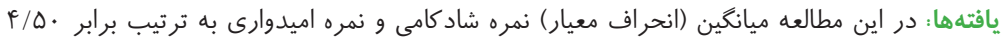

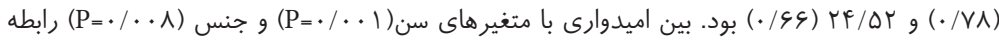

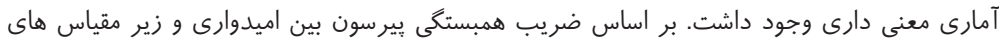

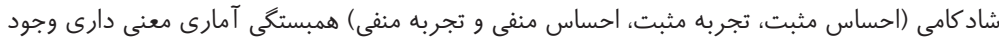

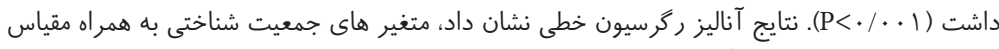

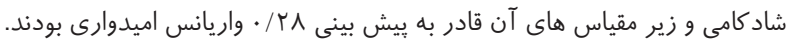

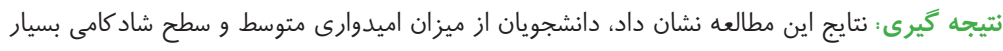

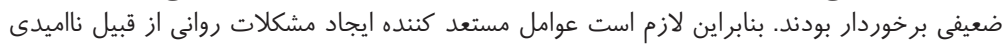

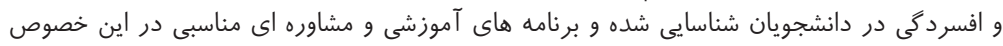

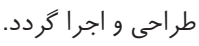

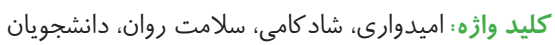

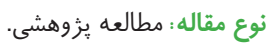

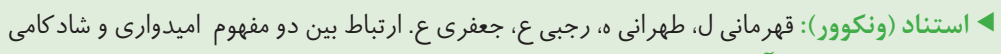

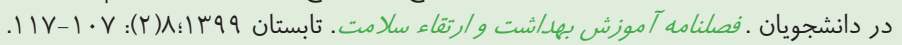

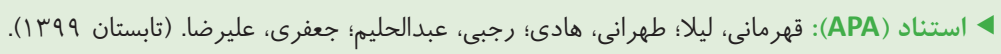

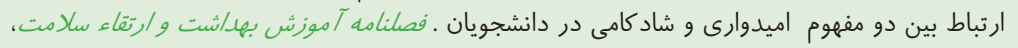

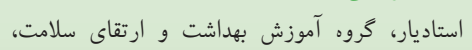

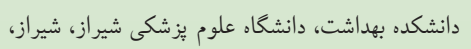

ايران.

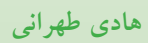

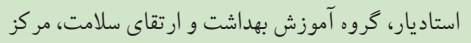

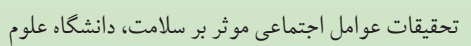
يزشكى مشهد، مشهد، ايران.

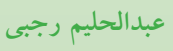

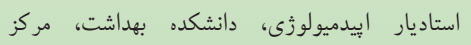

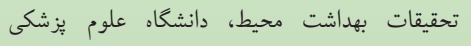
كلستان، كر كان، ايران.

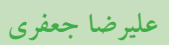
دانشجوى دكترى، مركز تحقيقات عوامل اجتماعى موثر بر سلامت، دانشكاء علوم بز شُشكى كناباد، كناباد، ايران.

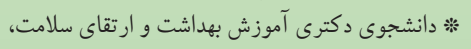

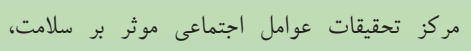
دانشكا، علوم يزشكى مشهد، مشهد، ايران.(نويسنده jafari.ar94@gmail.com

مسئول)

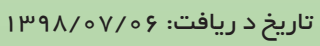

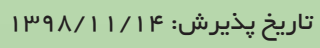


مطالعه ايى كه به طور سيستماتيك وضعيت مشكلات روانى را در مقدمه دانشجويان ايرانى مورد بررسى قرار داده بود، نشان داد كه بين

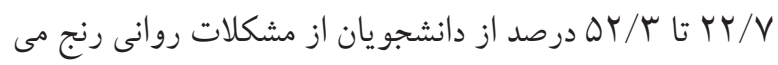

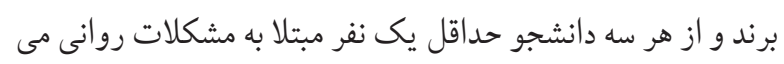

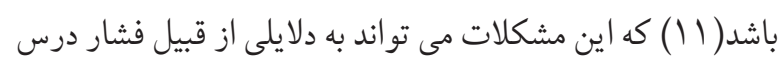

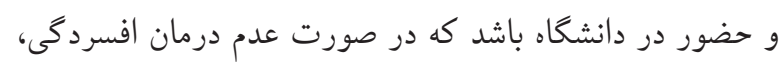

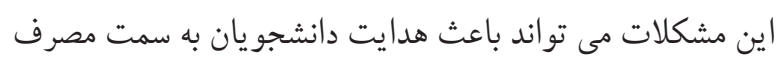

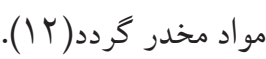

بر اساس نتايج مطالعات مختلف افزايش ميزان شادكامى باعث

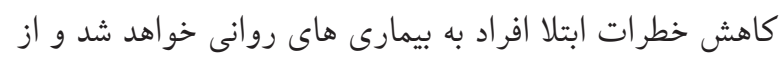

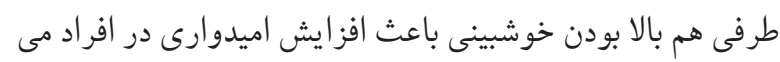

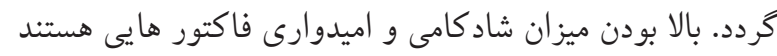
كه در ييشخيرى و كاهش مشكلات روانى در دانشجويان و افراد

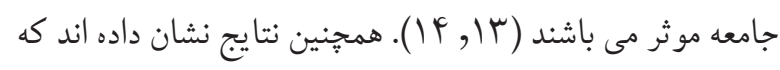

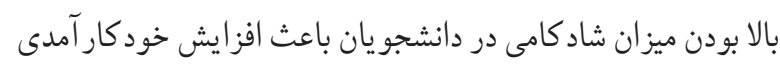

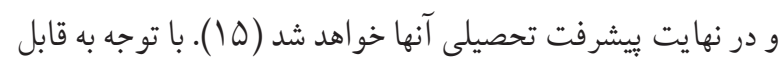

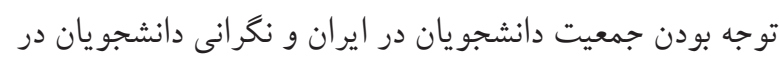

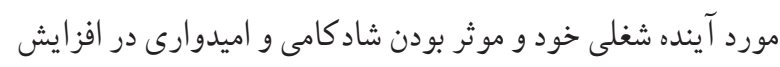

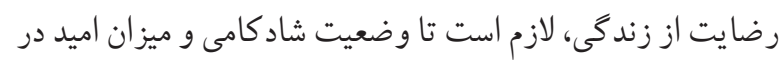

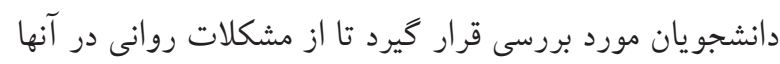

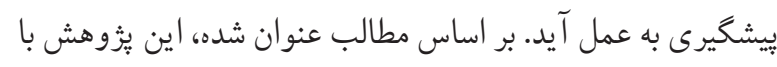

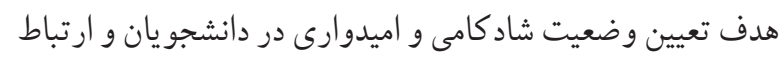
بين اين دو مفهوم انجام شده است.

مو اد و روش ها يُزوهش حاضر، يك مطالعه مقطعى از نوع توصيفى- تحليلى است

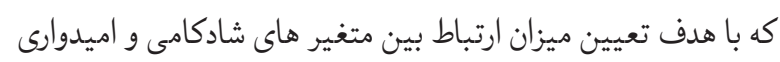

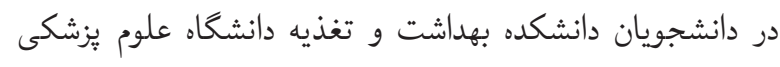
شيراز انجام شده است. دانشجوى مقطع كارشناسى بودن، كذراندن حداقل يك ترم از كل دوره تحصيل، اشتغال به تحصيل در ترم

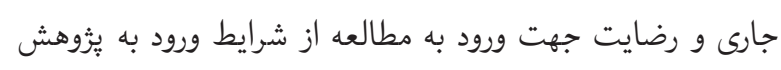

يكى از موضوعاتى كه در رويكردهاى روانشناسى مورد توجه قرار

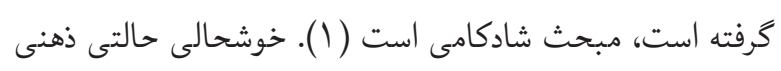

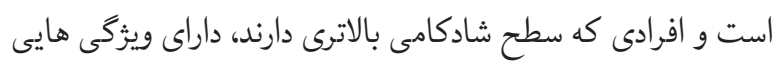
از قبيل اجتماعى بودن، احساس صلاحيت و شايستكى داشتن،

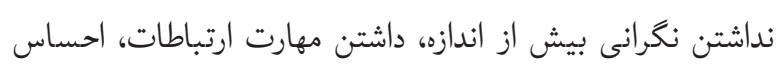

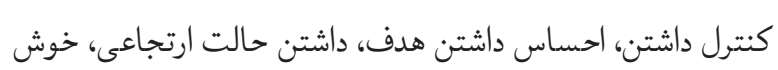
بينى، توجه به ظاهر خود، شوخ طبعى، تفريح و بازى و دورى از

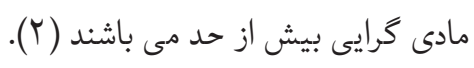

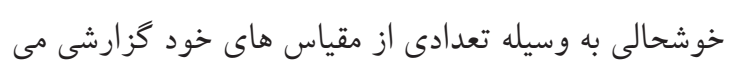

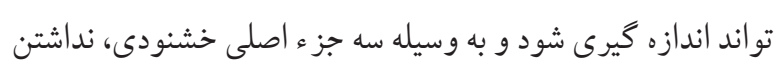
اضطر اب و احساسات مثبت مشخص مى شود (r, †). وقتى انسان خوشحال است دنيا را دليذير تر مى يابد، آسان تر و بهتر تصميم

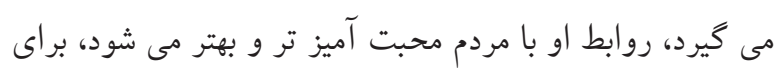
كمك به ديكران آماد گى بيشترى بيدا مى كند، اعتماد به خو يشتن

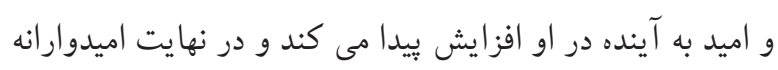

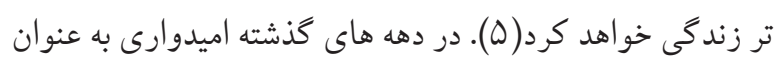
يك ساختار روانشناسى تحت بررسى دقيقى توسط محققين علاقمند

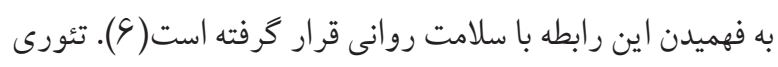

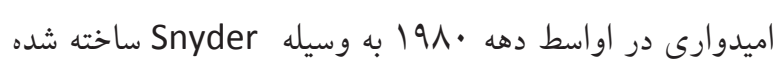

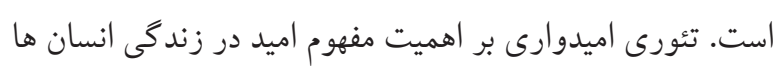
تاكيد كرده است (V) (V).

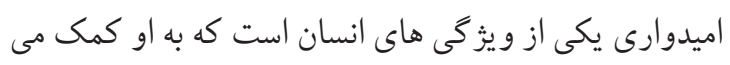

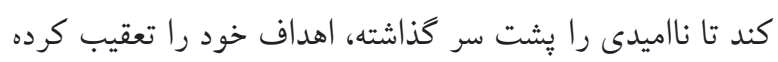
و احساس غير قابل تحمل بودن آينده را كاهش دهد (^). اين كند مفهومى است كه با سلامت جسمى، ذهنى و اندازه كيرى همزمان و ييش بينى مداخلات بزشكى، سلامت ذهنى، شوخ طبعى، دورى

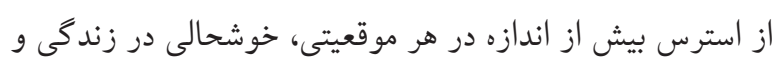
توانايى حل مشكل رابطه مستقيم دارد (9).

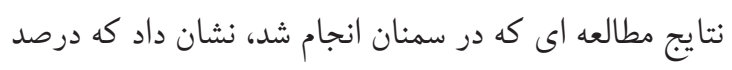

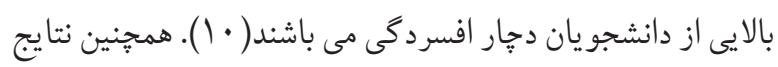




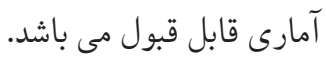

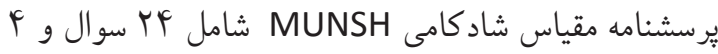
زير مقياس مى باشد كه با جمع كردن نمرات بر طبق كليد پاسخ

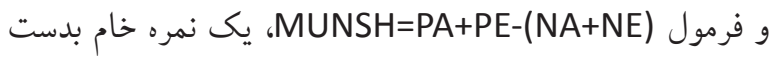
مى آيد. ه سوال براى سنجش زير مقياس احساس مثبت (PA)،

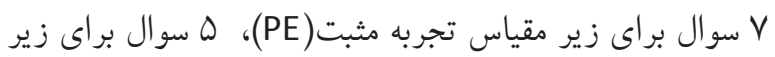
مقياس احساس منفى (NA) و Vو لوال براى زير مقياس تجربه

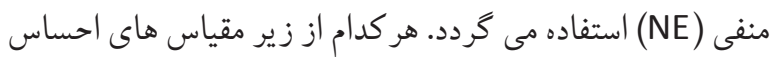
مثبت و منفى با داشتن ه سوال، نمره اى از صفر تا • ا و هر كدام

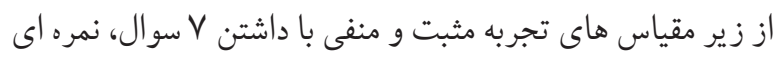

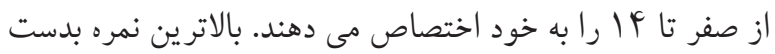

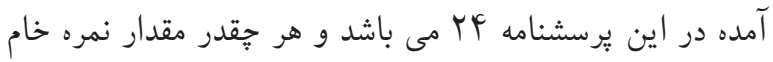
كسب شده بالاتر باشد، فرد داراى وضعيت روانشناختى بهترى

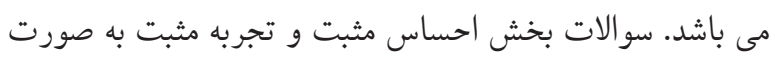

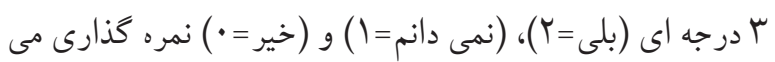

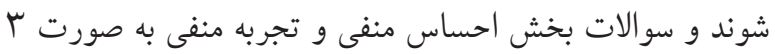

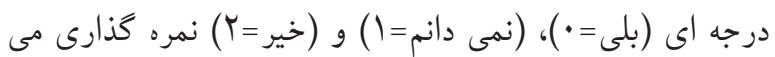
شوند. پايايى اين آزمون در مطالعات مختلف سنجيده شده است.

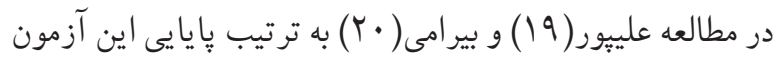

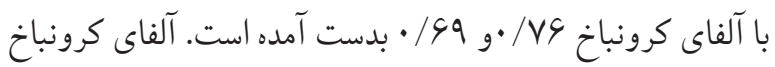

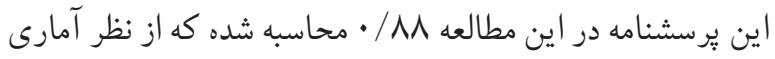
قابل قبول مى باشد.

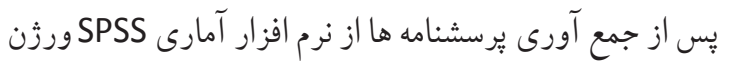

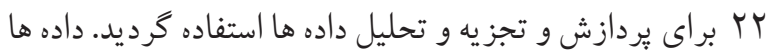
با استفاده از شاخص هاى ميانگين و انحر اف معيار توصيف شدند. براى مقايسه ارتباط بين متغير هاى جمعيت شناختى به صورت كيفى دو حالتى بازير مقياس هاى شاد كامى و اميدوارى از آزمون

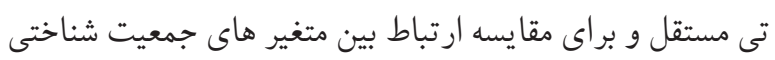

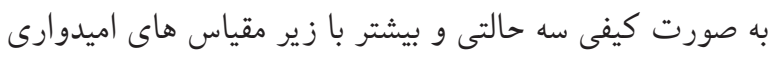
و شادكامى از آزمون آناليز وارياس (ANOVA) استفاده كرديد. براى بررسى ارتباط بين مقياس هاى شادكامى، اميدوارى و زير إنى
حاضر بود. جامعه آمارى اين مطالعه كليه دانشجويان مشغول به تحصيل خوابخاهى و غيرخوابگاهى در دانشكده بهداشت دانشگاه علوم يزشكى شيراز بود. براساس مطالعات قبلى(10) و با لحاظ

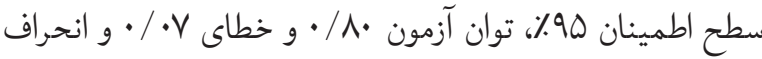
معيار OV / •، حجم نمونه مورد نياز بر اساس فرمول تخمين ميانگين r r نفر برآورد گرديد. نمونه هاى مطالعه به روش تصادفى ساده از بين دانشجو يانى تردي كه واجد شرايط ورود به مطالعه بودند، انتخاب شدند. لازم به ذكر است برسشنامه هاى افر ادى كه داراى اطلاعات ناقص بود از

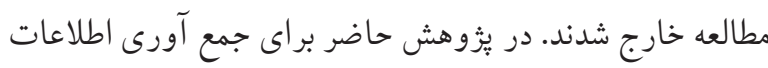
از يرسشنامه هاى اميدوارى Snyder و شادكامى MUNSH استفاده كرديد.

يرسشنامه مقياس اميدوارى Snyder و همكاران(

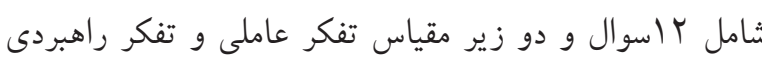

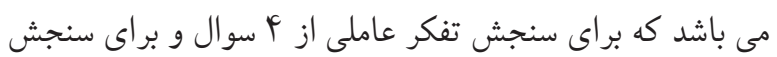
تفكر راهبردى از F سوال استفاده مى شود و F سوال باقى مانده

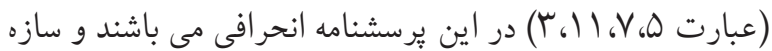

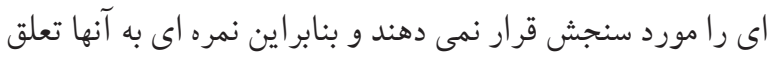

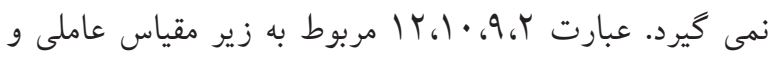

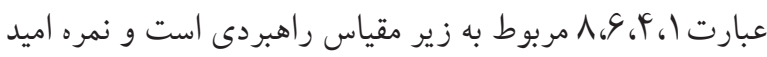

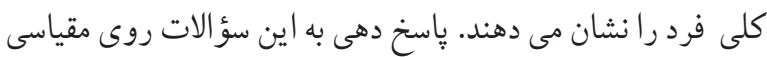

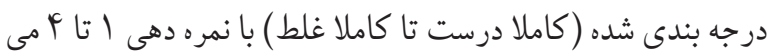

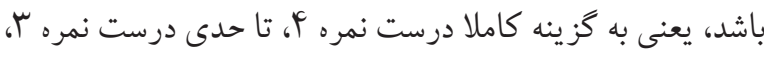

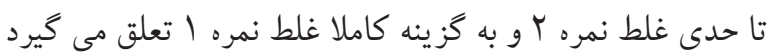

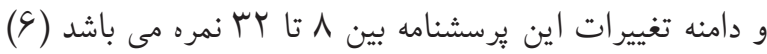

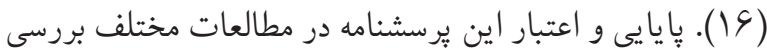

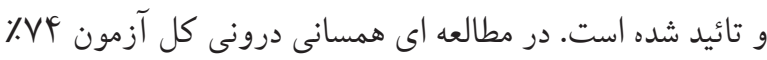

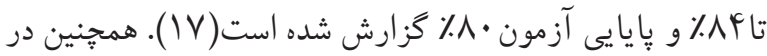

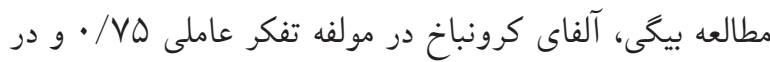

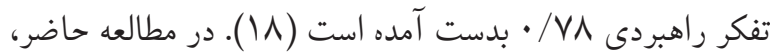

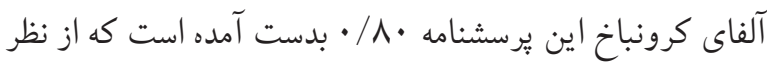


مقياسهاى آنها با يكديكر از آزمون همبستكى يِيرسون استفاده يزوهشكر محرمانه باقى خواهد ماند.

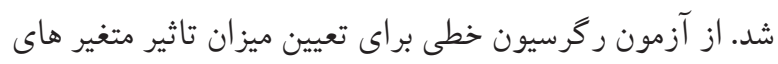

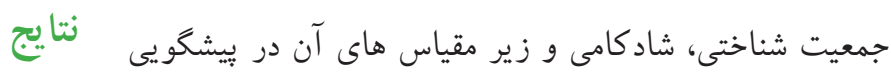

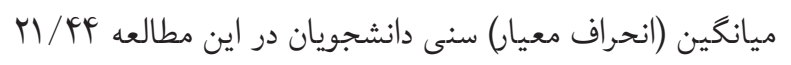
ميزان اميدوارى در دانشجويان استفاده كرديد.

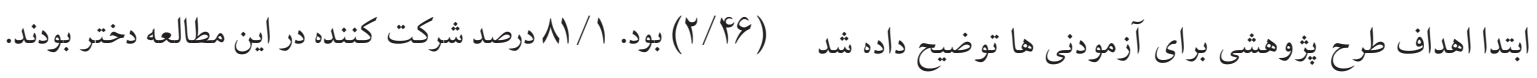

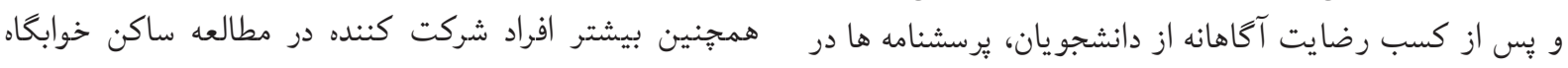

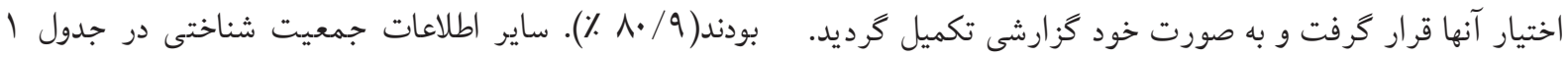

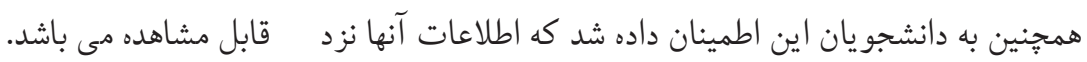
جدول ا: مقايسه ميانگين نمرات شاد كامى، اميدوارى و زير مقياس هاى آنها بر اساس متغير هاى دمو كرافيكى

\begin{tabular}{|c|c|c|c|c|c|c|c|c|c|c|}
\hline \multirow{2}{*}{ فراوانى (درصد) } & \multicolumn{3}{|c|}{ 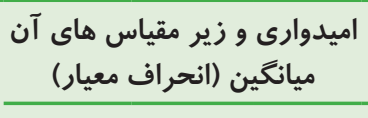 } & \multicolumn{5}{|c|}{ 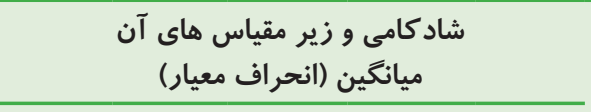 } & \multirow{2}{*}{\multicolumn{2}{|c|}{ متغير ها }} \\
\hline & اميدوارى & راهبردى تفكر & عاملى & شاد كامى & تجربه & احساس & تجربه & مثبت احساس & & \\
\hline $\begin{array}{c}191 \\
(11 / 1)\end{array}$ & $\begin{array}{l}r F / Y F \\
(r / G \cdot)\end{array}$ & $\begin{array}{l}\mid r / F F \\
(1 / q r)\end{array}$ & $\begin{array}{l}11 / 1 . \\
(r / 11)\end{array}$ & $\begin{array}{l}\text { N/9G } \\
(s / 1 \cdot)\end{array}$ & $\begin{array}{l}\Delta / \& G \\
(f / \cdot \Delta)\end{array}$ & $\begin{array}{l}0 / 91 \\
(r / 9 r)\end{array}$ & $\begin{array}{l}V / V Y \\
(r / F F)\end{array}$ & $\begin{array}{l}r / 90 \\
(r / / r)\end{array}$ & 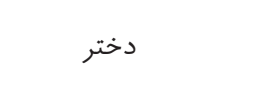 & \\
\hline $\begin{array}{c}\text { FG } \\
(11 / 9)\end{array}$ & $\begin{array}{l}r \Delta / \wedge F \\
(r / \wedge \mu)\end{array}$ & $\begin{array}{l}\mid r / r . \\
(1 / \wedge \gamma)\end{array}$ & $\begin{array}{l}\mid r / G F \\
(r / F \mid)\end{array}$ & $\begin{array}{l}\wedge / \vee \wedge \\
(\varepsilon / 1 Y)\end{array}$ & $\begin{array}{l}F / r \mu \\
(r / r \Delta)\end{array}$ & $\begin{array}{l}f / f \mu \\
(r / l V)\end{array}$ & $\begin{array}{l}V / V V \\
(r / g F)\end{array}$ & $\begin{array}{l}r / V V \\
(r / \wedge q)\end{array}$ & 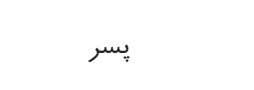 & جنس \\
\hline ----- & $\cdot / \cdots \wedge$ &.$/ \cdot 1 \mathrm{~V}$ &.$/ .11$ & . /Ast &.$/ \cdot r V$ &.$/ \mu$ &.$/ 9 r V$ & $\cdot / \mathrm{VHA}$ & معنى دارى" & \\
\hline $\begin{array}{c}190 \\
(1 \cdot / 9)\end{array}$ & $\begin{array}{l}r F / \Delta V \\
(r / \Delta I)\end{array}$ & $\begin{array}{l}1 r / 09 \\
(1 / \wedge 9)\end{array}$ & $\begin{array}{l}11 / 9 V \\
(r / .9)\end{array}$ & $\begin{array}{l}\wedge / \wedge \vee \\
(\Delta / \varsigma \subseteq)\end{array}$ & $\begin{array}{l}\Delta / r V \\
(r / \Lambda I)\end{array}$ & $\begin{array}{l}\Delta / \Delta r \\
(r / 9 q)\end{array}$ & $\begin{array}{l}V / V \cdot \\
(r / F \Delta)\end{array}$ & $\begin{array}{l}r / 9 \varepsilon \\
(r / \cdot 1)\end{array}$ & خوابعاه & \\
\hline $\begin{array}{c}\text { is } \\
(19 / 1)\end{array}$ & $\begin{array}{l}r F / V I \\
(F / r r)\end{array}$ & $\begin{array}{l}1 Y / 90 \\
(r / .9)\end{array}$ & $\begin{array}{l}\mid r / . G \\
(r / G \mid)\end{array}$ & $\begin{array}{l}9 / 10 \\
(V / 0 \cdot)\end{array}$ & $\begin{array}{l}\Delta / \Delta q \\
(f / F q)\end{array}$ & $\begin{array}{l}\Delta / 9 \wedge \\
(r / r \cdot)\end{array}$ & $\begin{array}{l}V / 9 G \\
(r / G \cdot)\end{array}$ & $\begin{array}{l}r / G \Lambda \\
(r / r V)\end{array}$ & غير خوابگاهى & محل سكونت \\
\hline ----- &.$/ 11$. & $\cdot / 1 \Delta S$ & $\cdot / \wedge \cdot \wedge$ & $\cdot / \mathrm{V} \wedge$. &.$/ 411$ & $\cdot / \mu V \mid$ &.$/ 900$ & $\cdot / \Delta \wedge \mathrm{V}$ & معنى دارى" & \\
\hline $\begin{array}{c}V V \\
(r / r)\end{array}$ & $\begin{array}{l}r \Psi / \Delta V \\
(r / V \varphi)\end{array}$ & $\begin{array}{l}\mid r / 9 \Lambda \\
(r / 11)\end{array}$ & $\begin{array}{l}11 / \lambda \Lambda \\
(r / \mid F)\end{array}$ & $\begin{array}{l}F / \Delta 1 \\
(r / r \mu)\end{array}$ & $\begin{array}{l}N / F\rangle \\
(r / 9 q)\end{array}$ & $\begin{array}{l}r / 1 q \\
(r / T F)\end{array}$ & $\begin{array}{l}V / \cdot G \\
(r / F F)\end{array}$ & $\begin{array}{l}F / V Y \\
(r / r F)\end{array}$ & بهداشت محيط & \\
\hline $\begin{array}{c}\wedge \varepsilon \\
(\Upsilon \vee / / 1)\end{array}$ & $\begin{array}{l}r \Delta / 1 . \\
(r / 19)\end{array}$ & $\begin{array}{l}1 r / V q \\
(1 / \Delta \Delta)\end{array}$ & $\begin{array}{l}\mid r / r . \\
(r / \cdot \Lambda)\end{array}$ & $\begin{array}{l}r / \mu F \\
(r / \Delta q)\end{array}$ & $\begin{array}{l}9 / \cdot 1 \\
(r / \wedge \vee)\end{array}$ & $\begin{array}{l}\varphi / \Delta G \\
(r / \cdot r)\end{array}$ & $\begin{array}{l}\Lambda / \mathcal{F} \cdot \\
(r / \vee \cdot)\end{array}$ & $\begin{array}{l}\Delta / \Delta F \\
(r / q \cdot)\end{array}$ & بهداشت عمومى & \\
\hline $\begin{array}{l}9 \Delta \\
(r \Lambda)\end{array}$ & $\begin{array}{l}r \mu / q \mu \\
(\mathcal{F} / r \mu)\end{array}$ & $\begin{array}{l}1 r / r q \\
(/ 19)\end{array}$ & $\begin{array}{l}11 / q F \\
(r / F T)\end{array}$ & $\begin{array}{l}f / \Delta G \\
(f / \mid r)\end{array}$ & $\begin{array}{l}N / F \\
(r / q r)\end{array}$ & $\begin{array}{l}r / 10 \\
(r / \wedge \Delta)\end{array}$ & $\begin{array}{l}V / s) \\
(r / .9)\end{array}$ & $\begin{array}{l}r / \Delta . \\
(r / \wedge s)\end{array}$ & بهداشت حرفه اى & رشته تحصيلى \\
\hline $\begin{array}{c}r \\
(1 / V)\end{array}$ & $\begin{array}{l}r Y / \Lambda \Lambda \\
(r / \Delta V)\end{array}$ & $\begin{array}{l}11 / q 4 \\
(1 / r q)\end{array}$ & $\begin{array}{l}11 / r r \\
(r / r \mid)\end{array}$ & $\begin{array}{l}F / V \Delta \\
(\Lambda V / Y)\end{array}$ & $\begin{array}{l}\wedge / \vee \Delta \\
(\kappa / \wedge \varsigma)\end{array}$ & $\begin{array}{l}r / V \Delta \\
(1 / \vee \cdot)\end{array}$ & $\begin{array}{l}V / \cdot q \\
(f / F F)\end{array}$ & $(\mathcal{F} / \mathrm{VS}) \Delta$ & تغذيه & \\
\hline -------- & $\cdot / r 11$ & $\cdot / r \cdot r$ & $\cdot / r \& \Lambda$ &.$/ 91 \mathrm{r}$ & $\cdot / 0 \cdot V$ & $\cdot / \vee 91$ & .1 .94 & $\cdot / \mathrm{IVr}$ & معنى دارى & \\
\hline
\end{tabular}

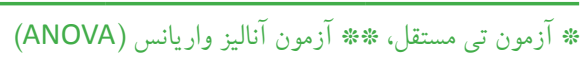

در اين مطالعه ميانگين (انحر اف معيار) نمره شاد كامى و نمره برقرار نبود. اما بين زير مقياس هاى احساس منفى و تجربه منفى با

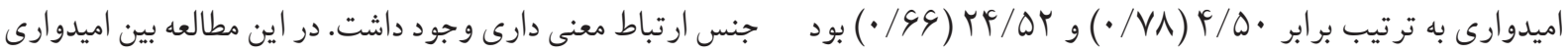

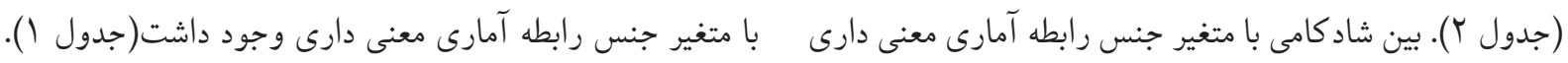




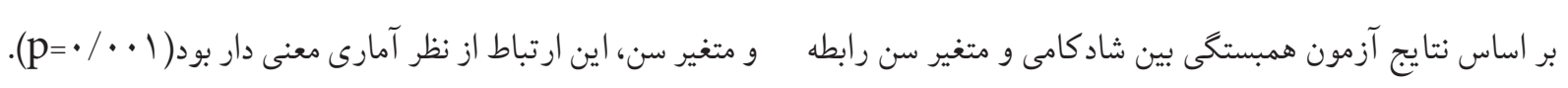

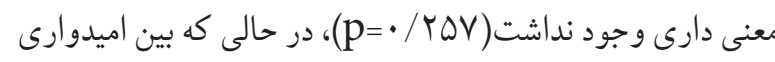

جدول r: نمرات ميانكَين (انحر اف معيار) و ضرايب آلفا كرونباخ متغيرهاى شادكامى، اميدوارى و زير مقياس هاى آنها

\begin{tabular}{|c|c|c|c|c|c|c|}
\hline بيشينه & كمينه & آلفاى كرونباخ & انحراف معيار & ميانكين & متغير ها & \\
\hline 1. & . & $\cdot /$ Y & $\cdot / \Lambda \Lambda$. & $r / 9 F$ & زير مقياس احساس مثبت(PA) & \multirow{5}{*}{ زير مقياس هاى شاد كامى } \\
\hline if & . & $\cdot / \mathrm{VI}$ & $\cdot / \mathrm{VS}$ & V/VQ & زير مقياس تجربه مثبت(PE) & \\
\hline 1. & . & $\cdot / \mathrm{V}$ & $\cdot / \mathrm{VI}$ & $r / \mu q$ & زير مقياس احساس منفى(NA) & \\
\hline If & . & $\cdot / \vee \wedge$ & $\cdot / \mathrm{V}$ & $\Lambda / \Delta S$ & زير مقياس تجربه منفى(NE) & \\
\hline$\varphi \wedge$ & . & $\cdot / \Lambda \Lambda$ & $\cdot / \mathrm{V} \Lambda$ & $F / 0$ & نمره كل شاد كامى & \\
\hline 19 & r & $\cdot / \mathrm{VI}$ & $\cdot / \Lambda$ & $11 / 90$ & تفكر عاملى & \multirow{3}{*}{ زير مقياس هاى اميدوارى } \\
\hline 19 & r & .194 & $\cdot / \mathrm{VI}$ & $\mid r / \Delta V$ & ت تفكر راهبردى & \\
\hline rt & $\wedge$ & $\cdot / \Lambda$ &.$/ 94$ & TF/Or & نمره كل اميدوارى & \\
\hline
\end{tabular}

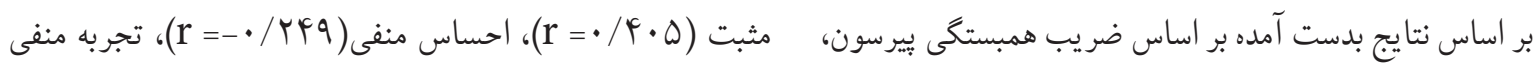

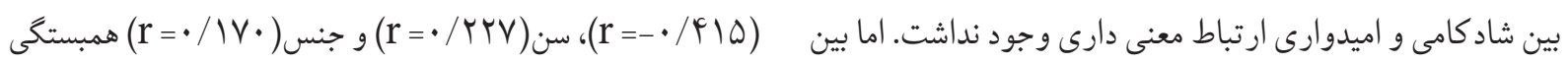

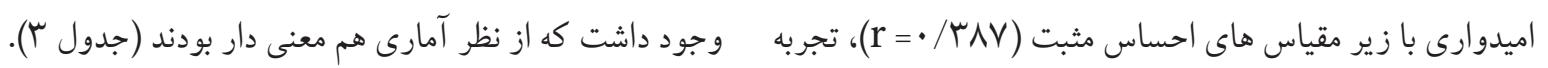
جدول سا: همبستخى ييرسون بين متغير هاى دموَّرافيك، شادكامى، اميدوارى و زير مقياس هاى آنها

\begin{tabular}{|c|c|c|c|c|c|c|c|c|c|}
\hline 9 & $\wedge$ & $\checkmark$ & 9 & $\Delta$ & f & $r$ & $r$ & 1 & متغير ها \\
\hline & & & & & & & & 1 & 1 - شاد كامى \\
\hline & & & & & & & 1 & $\begin{array}{c}\mathrm{p}=\cdot / 4 \mathrm{~V} \mid \\
-\cdot / \cdot \mathrm{rV}\end{array}$ & r- احساس مثبت \\
\hline & & & & & & 1 & $\begin{array}{c}\cdot / \Delta \vee \Delta \\
\mathrm{p}=\cdot / \cdot / 1\end{array}$ & $\begin{array}{l}-\cdot / \cdot F V \\
\mathrm{p}=\cdot / \kappa q .\end{array}$ & r- تجربه مثبت \\
\hline & & & & & 1 & $\begin{array}{c}\cdot / \text { א } \mathrm{c} \\
\mathrm{p}=\cdot / \cdot \text { l }\end{array}$ & 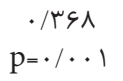 & $\begin{array}{c}\cdot / \cdot q r \\
\mathrm{p}=\cdot / \Delta \cdot q\end{array}$ & r ا احساس منفى \\
\hline & & & & 1 & $\begin{array}{c}\cdot / \Delta q . \\
p=\cdot / / 1\end{array}$ & $\begin{array}{c}\cdot / 0 r q \\
p=. / .1\end{array}$ & $\begin{array}{c}\cdot / f \Delta \cdot \\
\mathrm{p}=\cdot / \cdot / 1\end{array}$ & $\begin{array}{l}-\cdot / \cdot \mu r \\
\mathrm{p}=\cdot / \varphi^{\mu} \mid r\end{array}$ & ه- تجربه منفى \\
\hline & & & 1 & $\begin{array}{c}\cdot / 410 \\
\mathrm{p}=\cdot / \cdot \cdot 1\end{array}$ & $\begin{array}{c}\cdot / r F q \\
p=\cdot / \cdot 1\end{array}$ & $\begin{array}{c}\cdot / \mu \cdot \Delta \\
\mathrm{p}=\cdot / \cdot \cdot 1\end{array}$ & 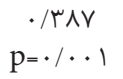 & $\begin{array}{l}-. / .99 \\
p=. / 11 V\end{array}$ & צ- اميدوارى \\
\hline & & 1 & $\begin{array}{c}\cdot / 9 \cdot 1 \\
\mathrm{p}=\cdot / \cdot \cdot 1\end{array}$ & $\begin{array}{c}\cdot / 410 \\
\mathrm{p}=\cdot / \cdot \cdot 1\end{array}$ & $\begin{array}{c}\cdot / r q \vee \\
\mathrm{p}=\cdot / \cdot \cdot 1\end{array}$ & $\begin{array}{c}\cdot / \not \leftarrow \wedge \\
\mathrm{p}=\cdot / \cdot / 1\end{array}$ & $\begin{array}{c}\cdot / \mathcal{f} \cdot \wedge \\
\mathrm{p}=\cdot / \cdot \cdot 1\end{array}$ & $\begin{array}{c}-\cdot / \cdot 1 \vee \vee \\
\mathrm{p}=\cdot / 199\end{array}$ & V - تفكر عاملى - \\
\hline & 1 & $\begin{array}{c}\cdot / 4 \cdots \\
p=\cdot / \cdots \mid\end{array}$ & $\begin{array}{c}\cdot / \wedge \Lambda \\
p=\cdot / \cdot / 1\end{array}$ & $\begin{array}{c}\cdot / \mu r T \\
p=\cdot / \cdot /\end{array}$ & $\begin{array}{c}. / / r q \\
p=. / . r \Lambda\end{array}$ & $\begin{array}{c}\cdot / r \& 4 \\
p=\cdot / \cdot 1\end{array}$ & $\begin{array}{c}\cdot / r \vee s \\
p=\cdot / / .1\end{array}$ & $\begin{array}{l}-. / .91 \\
p=. / 1 \Delta r\end{array}$ & ^-تفكر راهبردى \\
\hline 1 & $\begin{array}{c}\cdot / 191 \\
p=\cdot / . r p\end{array}$ & $\begin{array}{c}\cdot / r \mid F \\
p=\cdot / / . \mid\end{array}$ & $\begin{array}{c}\cdot / r r v \\
\mathrm{p}=\cdot / \cdot \cdot 1\end{array}$ & $\begin{array}{c}\cdot / 1.9 \\
p=. / 11 V\end{array}$ & $\begin{array}{c}. / 19 \\
p=. / .1 V\end{array}$ & $\begin{array}{c}\cdot / \cdot 19 \\
\mathrm{p}=\cdot / \mathrm{V} \Lambda\end{array}$ & $\begin{array}{c}\cdot / \cdot \wedge \cdot \\
\mathrm{p}=\cdot / \mu^{\mu r}\end{array}$ & $\begin{array}{c}\cdot / \cdot V q \\
p=\cdot / r \Delta V\end{array}$ & 9-9ن \\
\hline $\begin{array}{c}\cdot / / \wedge \Lambda \\
p=\cdot / \cdot \Delta\end{array}$ & $\begin{array}{c}. / 1 \Delta r \\
p=. / . / V\end{array}$ & $\begin{array}{c}. / 1 \Delta r \\
p=. / .1 \wedge\end{array}$ & $\begin{array}{c}\cdot / / \gamma \\
p=\cdot / / \wedge\end{array}$ & $\begin{array}{c}. / l F F \\
p=. / . r \Delta\end{array}$ & $\begin{array}{c}\cdot / 19 r \\
p=\cdot / \cdot r\end{array}$ & $\begin{array}{c}. / . \cdot 4 \\
\mathrm{p}=\cdot / 9 \mathrm{rV}\end{array}$ & $\begin{array}{l}-\cdot / \cdot r r \\
\mathrm{p}=\cdot / V Y \Lambda\end{array}$ & $\begin{array}{c}. / .90 \\
p=. / 4.9\end{array}$ & • 1 -جنس \\
\hline
\end{tabular}




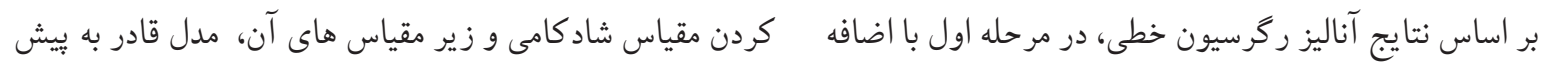

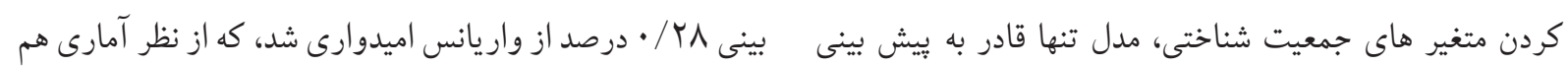

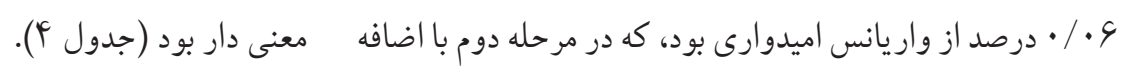
جدولأ: نتايج آناليز ركرسيون خطى در بيش بينى اميدوارى

\begin{tabular}{|c|c|c|c|c|c|c|c|c|}
\hline P-value & $\begin{array}{l}\text { Adjusted R } \\
\text { Square }\end{array}$ & P-value & $\mathbf{t}$ & 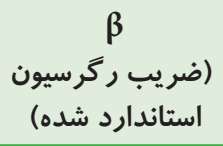 & (SE) B & $\begin{array}{c}\text { B } \\
\text { (ضريب رَاندارد نشده) }\end{array}$ & متغير & \\
\hline \multirow{4}{*}{$\cdot / \cdot r$} & \multirow{4}{*}{.1 .9} & $\cdot / \cdot r$ & $r / I V r$ & $\cdot / r \mid s$ & $\cdot / 1 \cdot r$ & ( & سن & \multirow{4}{*}{ مرحله اول } \\
\hline & & $\cdot / \cdot \wedge \Delta$ & $1 /$ Vrq &.$/ 111$ & . /srq & $1 / 1 \cdot r$ & جنس & \\
\hline & & $\cdot / \Lambda F V$ &.$- / 19 \pi$ &.$- / \cdot 1 \mu$ & $\cdot / s \mu F$ & سr/Ir & محل سكونت & \\
\hline & & $\cdot / I V r$ & $-1 / \mu \vee \cdot$ &.$- / .9 r$ & $\cdot / r 99$ & $-\cdot\left|F_{1}\right|$ & رشته تحصيلى & \\
\hline \multirow{9}{*}{$\cdot / \cdots 1$} & \multirow{9}{*}{$\cdot /$ / ^. } & $\cdot / \cdots r$ & r &.$/ 191$ & .1 .91 & $\cdot /$ YAD & سن & \multirow{9}{*}{ مرحله دوم } \\
\hline & & $\cdot / \cdot \sqrt{ } 1$ & $1 / \Lambda \mid r$ &.$/ 111$ & $\cdot / \Delta \wedge \vee$ & $1 / . G F$ & جنس & \\
\hline & & $\cdot / 9 r$ & $-\cdot / 1 \cdot 1$ & $-\cdot / \cdot c$ & $\cdot / \Delta S Y$ & $-\cdot / \cdot \Delta V$ & محل سكونت & \\
\hline & & $\cdot / 1 \wedge r$ & - & $-\cdot / \cdot \vee 9$ & $\cdot / r s \Delta$ & $-\cdot / r \Delta \Delta$ & رشته تحصيلى & \\
\hline & & $.1 .4 \wedge$ & I/Ars &.$/|f|$ &.$/ .94$ & $\cdot /|V|$ & احساس مثبت & \\
\hline & & $.1 \cdot 10$ & $r / \mathscr{F} \Delta 1$ &.$/ 19$ & •/.人 & $\cdot / r \cdot r$ & تجربه مثبت & \\
\hline & & $\cdot / M F F$ &.$- / 949$ & $-\cdot / \cdot \times 1$ & $.1 \cdot 9$ & $-\cdot / \cdot \wedge \varsigma$ & احساس منفى & \\
\hline & & $\cdot / \cdots 1$ & T/YrI & ./rGY & $\cdot / \cdot \sqrt{ } s$ & . TYSO & تجربه منفى & \\
\hline & & .1 .99 & $-1 /$ Arq & $-\cdot / 1 \cdot 9$ & $.1 \cdot 9$ & $\cdot / 11$ & شاد كامى & \\
\hline
\end{tabular}

جون در كير مسئوليت و كر فتارى هاى زندگى مى شوند، معمو لا از

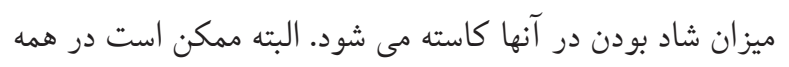
افراد اين موضوع صدق نكند و مسائل مختلفى تاثير كذار باشند. همجنين در اين مطالعه بين متغير جنسيت و اميدوارى رابطه

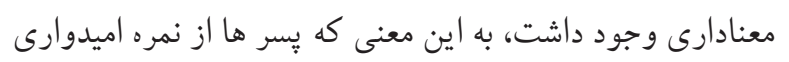

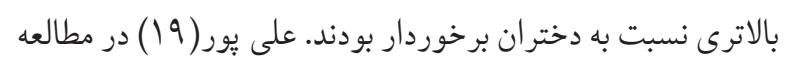

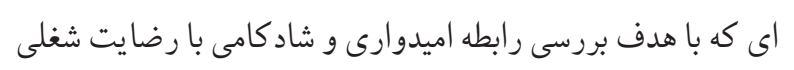

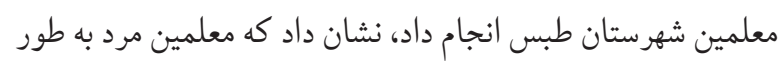

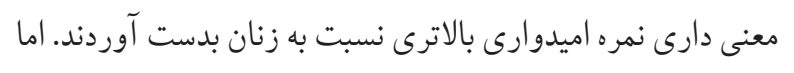
Creamer

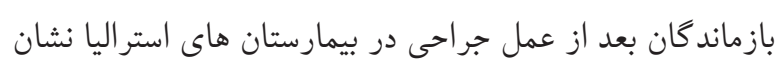

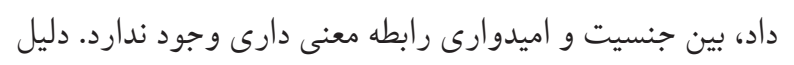

نتايج مطالعه حاضر نشان داد كه دانشجويان از ميزان اميدوارى متوسط و سطح شادكامى بسيار ضعيفى برخوردار مى باشند. ميانكين نمره

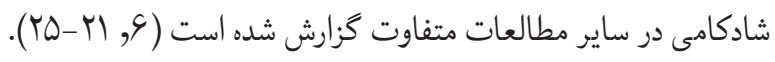

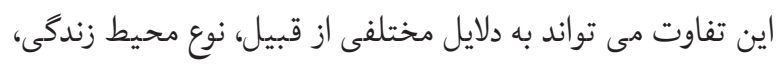

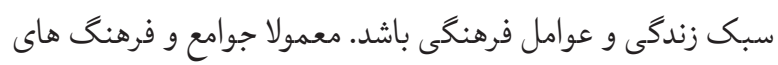
مختلف تعريف متفاوتى از شاد بودن و شاد زيستن دارند.

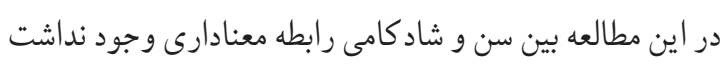

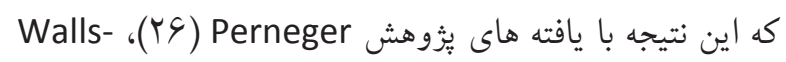
Ingram Shin

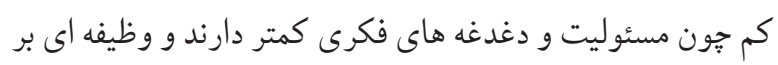

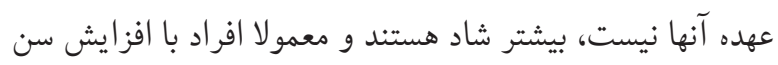




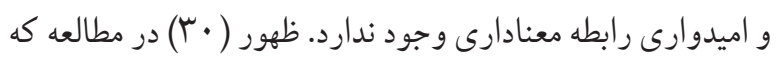
روى دانشجو يان انجام داد، نشان داد كه بين رشته هاى تحصيلى

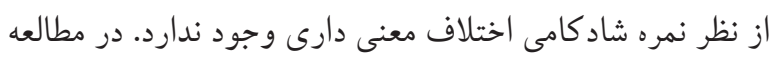

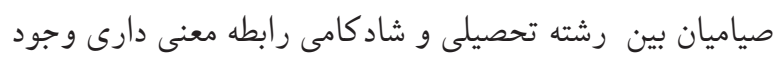

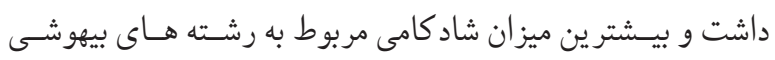

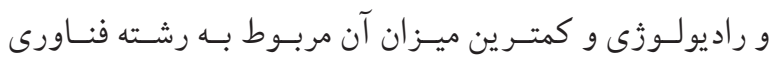

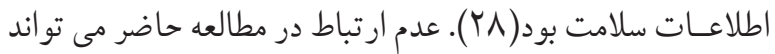
به دليل كافى نبودن تعداد رشته هاى تحصيلى باشد. معمولا مشاهده

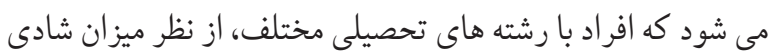

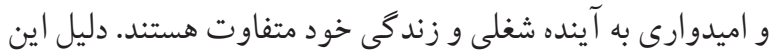

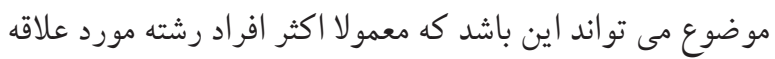

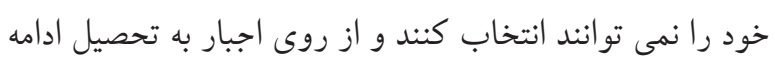
مى دهند و اين موضوع مى تو اند در ميزان شادى و اميدوارى آنها به آينده تاثير كذار باشد. بين ترم تحصيلى و شاخص شادكامى و اميدوارى هم رابطه معنادارى مشاهده نشد. در اكثر رشته هاى تحصيلى معمولا دانشجويان در ترم هاى اول به دليل ورود به دانشكاه خوشحال هستند و زياد

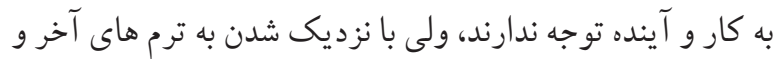

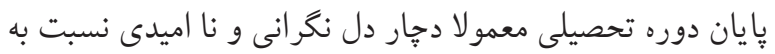

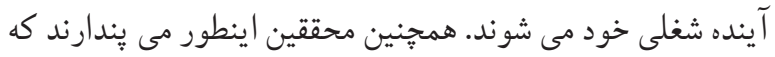

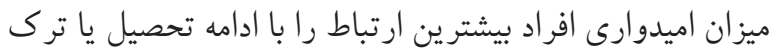

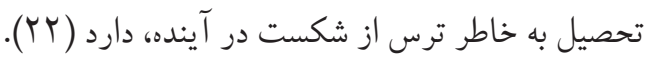

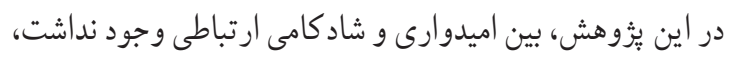

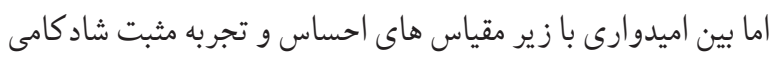
يك همبستكى متوسط مستقيم و با زير مقياس هاى احساس و و

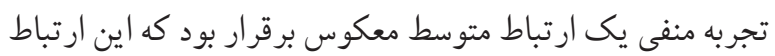

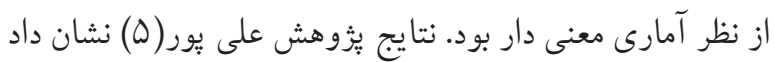
بين اميدوارى و شادكامى يك ارتباط مستقيم، قوى و معنى دار

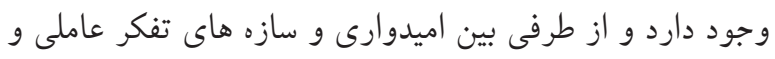
تفكر راهبردى يك ارتباط قوى، مستقيم و معنادارى وجود دارد.

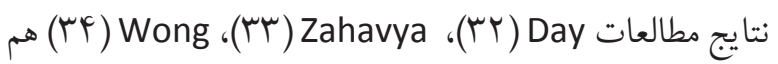

اين ناهمسو يى مى تواند به دليل متفاوت بودن گروه هاى هدف

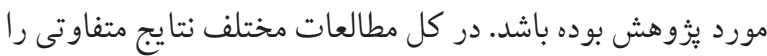
از لحاظ تاثير جنسيت در ميزان شادى افراد كزارش كرده اند. در بر برد حال حاضر به دليل مشغله هاى روزمره جدا از نوع جنسيت، افراد با مسائل مختلفى دركير هستند، همين دغدغه فكرى بخش زئه زيادى دوزي

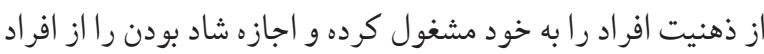
سلب كرده است. افراد با عدم نشخوار ذهنى و انجام فعاليت هاى مورد علاقه از قبيل انجام كارهاى هنرى، علمى و داشتن تفر يحات

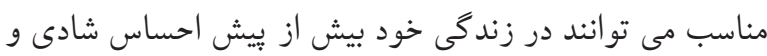
تندرستى كنند. با توجه به نسبت جمعيتى بيشتر دانشجو يان دختر

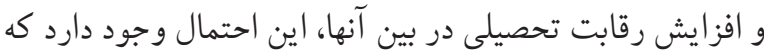

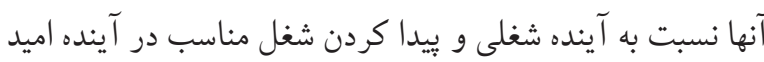
كم ترى داشته باشند.

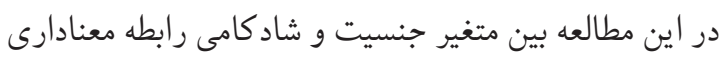
وجود نداشت. ظهور (·•) در مطالعه اي روى دانشجويان دانشكاه علوم يزشكى ايران نشان داد بين جنسيت و شاد كامى اختلاف معنى

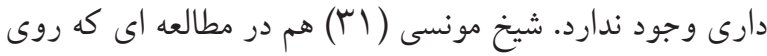

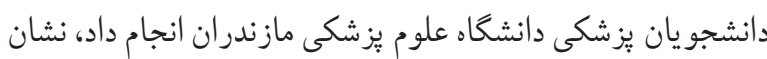
داد بين دو جنس تفاوت معنى دارى از نظر نمره شاد كامى وجود ندارد. يُّوهش حاضر نشان داد بين متغير محل سكونت با ميزان شاد كامى و اميدوارى رابطه معنادارى وجود ندارد كه اين با نتايج

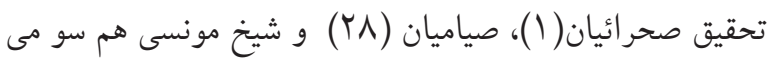
باشد. همجنين در اين مطالعه بين متغير محل سكونت با ميزان

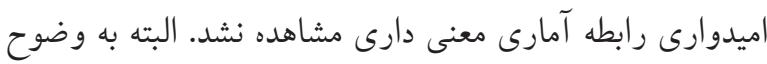

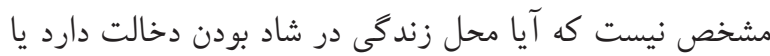
نه، بعضى ثزوهشها اين موضوع را اثبات و بعضى انكار كرده اند.

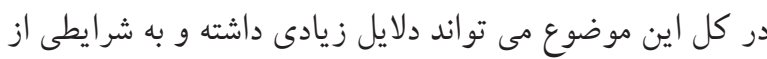

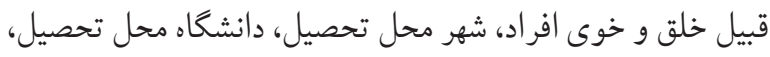
نوع خانواده و طبقه اجتماعى افراد مرتبط باشد و در شاد بودن فرد تاثير مثبت يا منفى داشته باشد.

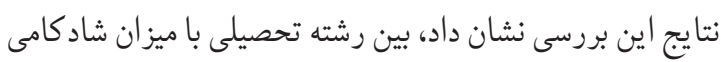


110
متفاوت با مطالعه حاضر بود كه مى تواند يك از دلايل متفاوت بودن نتايج آنها باشد. يكى از محدوديت هاى اين مطالعه عدم انجام

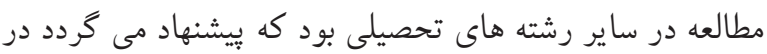
مطالعات آتى تمام رشته هاى تحصيلى مورد بررسى قرار گيرند. نتيجه كيرى: در نهايت نتايج اين مطالعه نشان مى دهد كه ميزان تهان شادكامى و اميدوارى در دانشجويان از سطح مناسبى برخوردار نبود. با توجه به نقش مهم موضوع اميدوارى و شاد كامى در زندگى تحصيلى و شخصى دانشجو يان لازم است مسئولين دانشخاه به اين مسئله توجه بيشترى كرده و عوامل مستعد كننده اى كه باعث ايجاد مشكلات روانى از قبيل ناميدى و افسردگى در دانشجويان

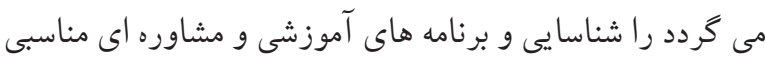
در اين خصوص طراحى و اجرا كنند. همجنين با طراحى و انجام

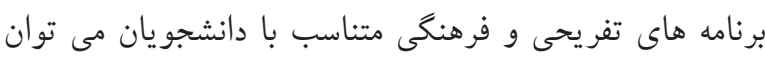

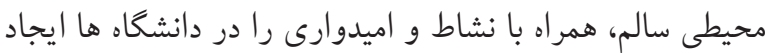
كرده و به ييشرفت تحصيلى دانشجويان كمك كرد.

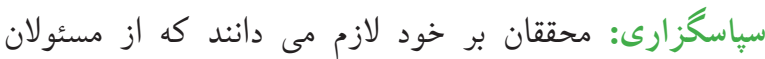
دانشكده بهداشت و تغذيه دانشخاه علوم يزشكى شيراز و تمام دانشجويان عزيزى كه ما را در انجام اين تحقيق يارى كرده اند، تقدير و قدردانى نمايند. تضاد منافع: نويسند گان مقاله هيج گونه تضاد منافعى درباره اين

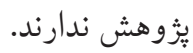

\section{References}

1. Sahraian A, Gholami A, Omidvar O. The Relationship Between Religious Attitude and Happiness in Medical Students in Shiraz University of Medical Sciences Ofogh-eDanesh. 2011;17(2):69-74.

2. Mozafarinia f, Shokravi FA, hydarnia A. Relationship between spiritual health and happiness among students. Iranian Journal of Health Education and Health Promotion. 2014;2(2):97-108.

3. Zabeti Arani M, Khodabakhshi-Kolaee A. The Effectiveness of Making and Collection of Family Albums on Autobiographical memory and Happiness in Elderly. Journal of Health Literacy. 2017;2(2):117-26. [DOI:10.22038/jhl.2017.10946]
تائيد كننده نتايج مطالعه حاضر بودند.

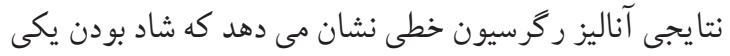
از فاكتور هاى مهمى است كه مى تواند در افزايش ميزان اميدوارى

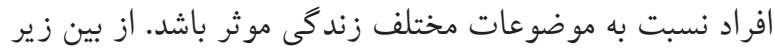

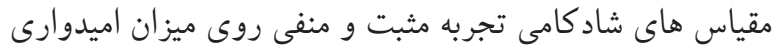

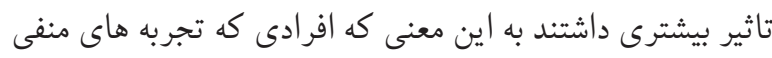
كمتر و تجربه هاى مثبت بيشترى در زندگى دارند، ميزان اميدوارى

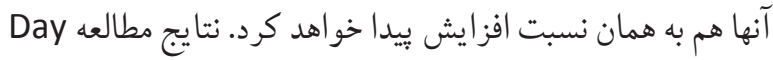
(Tr) كه با هدف بررسى تاثير اميدوارى روى بيشرفت تحصيلى، هوش و شخصيت دانشجويان دانشخاه هاى انگلستان انجام شده بود، متغير هاى جنس، سن، تفكر عاملى و تفكر راهبردى، تفكر واخرا، برونكرايى، وضعيت روانى، وفادارى و تجربه قادر به بيشكويى FF درصد واريانس مقياس اميدوارى بودند. در مطالعه اي كه Kemer

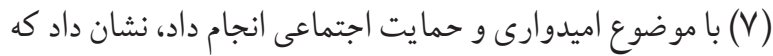
در نهايت متغير هاى يشتيبانى عاطفى، حمايت از عشق و احترام، يشتيبانى مرتبط با افشاى و وذيرش، پُشتيبانى اطلاعاتى، حمايت احساسى فعال و يشتيبانى ابزارى، جنسيت و محل اقامت قادر به يبشكو يیى الدرصد واريانس مقياس اميدوارى مى باشند. نتايج مطالعه كرمى كه روى بيماران بيمارستان هاى زاهدان انجام شده است(هץ) هم نشان داد، شوخ طبعى به طور معنى دارى آب درصد

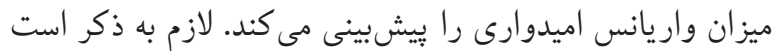
متغير هاى مورد استفاده در مدل رگرسيون خطى مطالعات ذكر شده،

4. Ladhari R, Souiden N, Dufour B. The role of emotions in utilitarian service settings: The effects of emotional satisfaction on product perception and behavioral intentions. Journal of Retailing and Consumer Services. 2017;34:10-8. [DOI:10.1016/j.jretconser.2016.09.005]

5. Alipour A, Aerab Sheibani K. Relation of Hopeness and Happiness with Job Satisfaction among Teachers. Journal of Psychology. 2011;6(22):71-84.

6. Creamer M, O'Donnell ML, Carboon I, Lewis V, Densley K, McFarlane A, et al. Evaluation of the Dispositional Hope Scale in injury survivors. Journal of Research in Personality. 2009;43(4):613-7. [DOI:10.1016/j.jrp.2009.03.002]

7. Kemer G, Atik G. Hope and Social Support in High School 
Students from Urban and Rural Areas of Ankara, Turkey. Journal of Happiness Studies. 2011;13(5):901-11. [DOI:10.1007/s10902-011-9297-z]

8. Rassouli M, Yaghmaie F, Alavi Majd H. Psychometric properties of "Hopefulness Scale for Adolescents" in Iranian institutionalized adolescents Payesh. 2010;9(2):197-204.

9. Hariri M, Khodami N. A study of the efficacy of teaching happiness Based on the Fordyce method to elderly people on their life expectancy. Procedia - Social and Behavioral Sciences. 2011; 30:1412-5. [DOI:10.1016/j. sbspro.2011.10.275]

10. Mahshid Bahrami M, Dehdashti A, karami M. Investigation Depression Prevalence and Related Effective Factors Among Students at Health Faculty Semnan University of Medical Sciences in 2017, Iran J Zanko Journal of Medical Sciences. 2017;18(58):24-32.

11. Alizadeh-Navaei R, Hosseini SHJCE. Mental health status of Iranian students until 2011: A systematic review. Journal of Clinical Excellence. 2014;2(1):1-10.

12. Pedrelli P, Nyer M, Yeung A, Zulauf C, Wilens T. College students: mental health problems and treatment considerations. Academic Psychiatry. 2015;39(5):503-11. [DOI:10.1007/s40596-014-0205-9] [PMID] [PMCID]

13. Conversano C, Rotondo A, Lensi E, Della Vista O, Arpone F, Reda MA. Optimism and its impact on mental and physical well-being. Clinical practice and epidemiology in mental health: CP \& EMH. 2010;6:25. [DOI:10.2174/1745017901 006010025] [PMID] [PMCID]

14. Griggs S. Hope and mental health in young adult college students: an integrative review. Journal of psychosocial nursing and mental health services. 2017;55(2):28-35. [DOI:10.3928/02793695-20170210-04] [PMID]

15. Saffari $M$, Sanaeinasab $H$, Rashidi Jahan $H$, Purtaghi G, Pakpour A. Happiness, Self-efficacy and Academic Achievement among Students of Baqiyatallah University of Medical Sciences. Journal of Medical Education Development. 2014;7(13):45-56.

16. Babyak MA, Snyder CR, Yoshinobu L. Psychometric properties of the hope scale: A confirmatory factor analysis. Journal of research in personality 1993; 27:15467. [DOI:10.1006/jrpe.1993.1011]

17. Maki AA. The Relationship between Spirituality and Successful Aging Older Minority Women. The Graduate School: The Florida State University 2005.

18. Beigi A, Farahani M, Mohammad-Khani S, MohammadiFar M. The Discriminative Role of Quality of Life and Hope in Narcotic Anonymous and Methadone Maintenance Groups. Journal of Clinical Psychology 2011;3(3):75-84.
[DOI:10.22075/JCP.2017.2064]

19. Alipour A, Hashemi T, Babapour J, Tousi F. Relationship between Coping Strategies and Happiness among University Students. Journal of Psychology 2010;5(11):7589.

20. Bayrami M, Toraj H, Parvaneh A, Ansar Vahideh A. Demographic factors, relying on the Islamic religion and family functioning in prediction of students'happiness in Tabriz University of Medical Sciences, Iran Psychology and Educational Sciences. 2010;9(1):33-43.

21. Barati F, Ashrafi Z, Najafi F, Karimi A, Sharifzadeh G. The Investigation Of Happiness Status And Its Related Factors In Students Of Birjand University Of Medical Sciences In 2013. Journal of Torbat Heydariyeh University of Medical Sciences. 2015;3(2):26-30.

22. Alexander ES, Onwuegbuzie AJ. Academic procrastination and the role of hope as a coping strategy. Personality and Individual Differences. 2007;42(7):1301-10. [DOI:10.1016/j.paid.2006.10.008]

23. Garossi MT, Mani A, Bakhshipour A. The relationship between personality characteristics and happiness feeling among students of Tabriz University. Journal of Psychology. 2006;1(1):144-57.

24. Ghanbarian M, Parmouz M, Rajabzadeh Z. The relationship between the happiness and life expectancy among the students of medical sciences in Behbahan city. Journal of Fundamentals of Mental Health. 2016;18(Special Issue):553-6. [DOI:10.22038/JFMH.2016.8509]

25. Sheng Y, Qiu Z-Q, He Y, Juniper N, Zhang Y. Living Conditions and Palliative Care Needs among End-of-Life Former Commercial Plasma Donors Affected with HIV/AIDS in Rural Henan of China. Biomedical and Environmental Sciences. 2010;23(4):279-86. [DOI:10.1016/S08953988(10)60064-5]

26. Perneger TV, Hudelson PM, Bovier PA. Health and happiness in young Swiss adults. Quality of Life Research 2004;13:1718. [DOI:10.1023/B:QURE.0000015314.97546.60] [PMID]

27. Walls-Ingram SA. The Relationship between Spirituality and Successful Aging Older Minority Women. Reutilization and quality of life University of Saskatchewan Saskatoon 2006.

28. Siamian H, Naeimi O, Shahrabi A, Hasanzadeh R. The Status of Happiness and its Association with Demographic Variables among the Paramedical Students J Mazand Univ Med Sci 2012;22(86):168-75.

29. Shin HS, Han HR, Kim MT. Predictors of psychological well-being amongst Korean immigrants to the United States: a structured interview survey. Int J Nurs Stud. 
2007;44(3):415-26. [DOI:10.1016/j.ijnurstu.2006.04.007] [PMID]

30. Zohoor A, Fekri A. Students' Happiness of School of Management and Medical Information Sciences Iran University of Medical Sciences and Health Services. Armaghane danesh. 2003;8(2):63-72.

31. Sheikhmoonesi F, Zarghami M, Khademloo $M$, Alimohammadi MM. Happiness and Associated Demographic Factors among Medical Students of Mazandaran University of Medical Sciences, 2010. Journal of Mazandaran University of Medical Sciences. 2013;22(97):132-7.

32. Day L, Hanson K, Maltby J, Proctor C, Wood A. Hope uniquely predicts objective academic achievement above intelligence, personality, and previous academic achievement. Journal of Research in Personality. 2010;44(4):550-3. [DOI:10.1016/j.jrp.2010.05.009]

33. Drach-Zahavya A, Somech A. Coping with health problems: the distinctive relationshipsof Hope subscales with constructive thinking and resource allocation. Personality and Individual Differences. 2002; 33:103-17. [DOI:10.1016/S0191-8869(01)00138-6]

34. Wong SS, Lim T. Hope versus optimism in Singaporean adolescents: Contributions to depression and life satisfaction. Personality and Individual Differences. 2009;46(5-6):648-52. [DOI:10.1016/j.paid.2009.01.009]

35. Karami A, Kahrazei F, Arab A. The role of humor in hope and posttraumatic growth among patients with leukemia. Journal of Fundamentals of Mental Health 2018;20(3):17684. 